\title{
Delayed choice for entanglement swapping
}

\author{
ASHER PERES \\ Department of Physics, \\ Technion-Israel Institute of Technology, \\ 32000 Haifa, Israel
}

\begin{abstract}
Two observers (Alice and Bob) independently prepare two sets of singlets. They test one particle of each singlet along an arbitrarily chosen direction and send the other particle to a third observer, Eve. At a later time, Eve performs joint tests on pairs of particles (one from Alice and one from Bob). According to Eve's choice of test and to her results, Alice and Bob can sort into subsets the samples that they have already tested, and they can verify that each subset behaves as if it consisted of entangled pairs of distant particles, that have never communicated in the past, even indirectly via other particles.
\end{abstract}

To appear in a special issue of Journal of Modern Optics. 


\section{Introduction and notations}

Since the early days of quantum mechanics, it has been known that after two quantum systems interact, their joint state is usually entangled and it may remain so even after these systems separate and are far away from each other. However, a direct interaction is not necessary in order to produce entanglement between distant systems. For example, any existing entanglement between two particles can be teleported to other, distant particles, by performing suitable joint measurements and broadcasting their results as classical information [1]. Protocols known as entanglement swapping have recently been proposed $[2,3]$ and experimentally realized [4]. In the present article, I propose an even more paradoxical experiment, where entanglement is produced a posteriori, after the entangled particles have been measured and may no longer exist.

To simplify the discourse and the notations, the experiment will be described in terms of spin- $\frac{1}{2}$ particles. (In the real world, it would be easier to use polarized photons. The spin sphere then has to be understood as a Poincaré sphere, and the argument is exactly the same.) The eigenstates of $\sigma_{z}$ will be denoted as

$$
\left(\begin{array}{l}
1 \\
0
\end{array}\right) \equiv|0\rangle \quad \text { and } \quad\left(\begin{array}{l}
0 \\
1
\end{array}\right) \equiv|1\rangle \text {. }
$$

In that basis, the other spin components have the following nonvanishing matrix elements:

$$
\left\langle 0\left|\sigma_{x}\right| 1\right\rangle=\left\langle 1\left|\sigma_{x}\right| 0\right\rangle=1, \quad\left\langle 0\left|\sigma_{y}\right| 1\right\rangle=-\left\langle 1\left|\sigma_{y}\right| 0\right\rangle=-i
$$

We shall also need the spin components along directions at $45^{\circ}$ from the $x$ and $y$ axes:

$$
\sigma_{ \pm}=\left(\sigma_{x} \pm \sigma_{y}\right) / \sqrt{2}
$$

These give

$$
\left\langle 0\left|\sigma_{ \pm}\right| 1\right\rangle=\left\langle 1\left|\sigma_{\mp}\right| 0\right\rangle=(1 \mp i) / \sqrt{2}
$$

For a pair of particles, it is convenient to define "Bell states" [5]

$$
\Phi^{ \pm}=(|00\rangle \pm|11\rangle) / \sqrt{2} \quad \text { and } \quad \Psi^{ \pm}=(|01\rangle \pm|10\rangle) / \sqrt{2} .
$$

Note that $\Psi^{-}$is the singlet state.

In the proposed experiment, two distant observers, conventionally called Alice and Bob, independently prepare two sets of singlets, whose states are denoted as $\Psi_{A}^{-}$and $\Psi_{B}^{-}$. 
Alice and Bob keep one particle of each singlet and send the other particle to a third observer, Eve, who also arranges them in pairs (one from Alice and one from Bob). The three observers keep records specifying to which pair each particle belongs.

The joint state of a pair of singlets can thus be written as

$$
\Psi_{A}^{-} \otimes \Psi_{B}^{-} \equiv\left(\Psi_{E}^{+} \otimes \Psi^{+}-\Psi_{E}^{-} \otimes \Psi^{-}-\Phi_{E}^{+} \otimes \Phi^{+}+\Phi_{E}^{-} \otimes \Phi^{-}\right) / 2
$$

where the subscript $E$ refers to the particles that are sent to Eve, and the symbols $\Phi^{ \pm}$and $\Psi^{ \pm}$without a subscript refer to the particles that Alice and Bob keep. Note the minus signs in Eq. (6). It would be possible to eliminate them by using, instead of the Bell basis, the "magic basis" $[6,7]$ where $\Phi^{-}$and $\Psi^{+}$are multiplied by $i$.

\section{Analysis of the results of measurements}

Alice and Bob now measure the values of spin components (along arbitrary directions) of the particles that they kept. For example, Alice measures spin component $\sigma_{x}$ or $\sigma_{y}$ (randomly chosen) of her particles, and likewise Bob measures $\sigma_{ \pm}$on his particles. These components were chosen because Alice and Bob want to test Bell inequalities, in the form given by Clauser, Horne, Shimony, and Holt (CHSH) [8]. The results that Alice and Bob get, namely \pm 1 , are of course completely random and uncorrelated.

At a later time, Eve performs joint tests on her pairs of particles. Just as in the teleportation scenario [1], she performs Bell measurements [5] and she informs the other observers of the results that she found. Using that information, Alice and Bob sort the

records of their measurements into four subsets, according to whether Eve found $\Phi^{ \pm}$or $\Psi^{ \pm}$. It then follows from Eq. (6) that, in each subset, the state of the particles that Alice and Bob kept was the same as the state later found by Eve. Thus, in each subset, there are nonvanishing expectation values $\left\langle\Phi^{ \pm}\left|\sigma_{a} \otimes \sigma_{b}\right| \Phi^{ \pm}\right\rangle$and $\left\langle\Psi^{ \pm}\left|\sigma_{a} \otimes \sigma_{b}\right| \Psi^{ \pm}\right\rangle$. Explicitly, owing to Eqs. (2) and (4), we have

$$
\begin{aligned}
\left\langle\Psi^{+}\left|\sigma_{x} \otimes \sigma_{ \pm}\right| \Psi^{+}\right\rangle & =-\left\langle\Psi^{-}\left|\sigma_{x} \otimes \sigma_{ \pm}\right| \Psi^{-}\right\rangle=1 / \sqrt{2} \\
\left\langle\Psi^{+}\left|\sigma_{y} \otimes \sigma_{ \pm}\right| \Psi^{+}\right\rangle & =-\left\langle\Psi^{-}\left|\sigma_{y} \otimes \sigma_{ \pm}\right| \Psi^{-}\right\rangle= \pm 1 / \sqrt{2} \\
\left\langle\Phi^{+}\left|\sigma_{x} \otimes \sigma_{ \pm}\right| \Phi^{+}\right\rangle & =-\left\langle\Phi^{-}\left|\sigma_{x} \otimes \sigma_{ \pm}\right| \Phi^{-}\right\rangle=1 / \sqrt{2} \\
\left\langle\Phi^{+}\left|\sigma_{y} \otimes \sigma_{ \pm}\right| \Phi^{+}\right\rangle & =-\left\langle\Phi^{-}\left|\sigma_{y} \otimes \sigma_{ \pm}\right| \Phi^{-}\right\rangle=\mp 1 / \sqrt{2}
\end{aligned}
$$


Thus, in each subset, one of the following CHSH inequalities is violated (and the others are satified):

$$
\begin{aligned}
& -2 \leq\left\langle\sigma_{x} \otimes \sigma_{+}+\sigma_{x} \otimes \sigma_{-}+\sigma_{y} \otimes \sigma_{+}-\sigma_{y} \otimes \sigma_{-}\right\rangle \geq 2, \\
& -2 \leq\left\langle\sigma_{x} \otimes \sigma_{+}+\sigma_{x} \otimes \sigma_{-}-\sigma_{y} \otimes \sigma_{+}+\sigma_{y} \otimes \sigma_{-}\right\rangle \geq 2 .
\end{aligned}
$$

For the subset associated with $\Psi_{E}^{-}$, it is the left hand side of Eq. (11) that is violated, for $\Psi_{E}^{+}$, it is the right hand side. For the subset associated with $\Phi_{E}^{-}$, it is the left hand side of Eq. (12) that is violated; and for $\Phi_{E}^{+}$, it is the right hand side. In other words, Alice and Bob find experimentally that each one of the four postselected subsets produces statistical results identical to those arising from maximally entangled pairs.

\section{The paradox}

There can be no doubt that the particles that were independently produced and tested by Alice and Bob were uncorrelated and therefore unentangled. Each one of these particles may well have disappeared (e.g., been absorbed) before the next particle was produced, and before Eve performed her tests. Only the records kept by the three observers remain, to be examined objectively.

How can the appearance of entanglement arise in these circumstances? The point is that it is meaningless to assert that two particles are entangled without specifying in which state they are entangled, just as it is meaningless to assert that a quantum system is in a pure state without specifying that state [9]. If this simple rule is forgotten, or if we attempt to attribute an objective meaning to the quantum state of a single system, curious paradoxes appear: quantum effects mimic not only instantaneous action-at-a-distance but also, as seen here, influence of future actions on past events, even after these events have been irrevocably recorded.

Note in particular that even after Alice and Bob have recorded the results of all their measurements, Eve still has the freedom of deciding which experiment she will perform. It can be a Bell measurement as proposed above, but the latter can also be preceded by

arbitrary bilateral rotations of the two spin- $\frac{1}{2}$ particles (this corresponds to an arbitrary real orthogonal transformation of the magic basis $[6,7])$. Eve can also perform an incomplete Bell measurement [10-12], or any other measurement she decides, represented by any positive operator valued measure (POVM) [9] of her choice. The only demand is that 
at least one of her outcomes corresponds to a definite entangled state of the other pair of particles, namely those retained by Alice and Bob (though not necessarily a maximally entangled state, nor even a pure state of these particles) [13].

Proof: Let $\mathbb{1}_{E}$ and $\mathbb{1}_{A B}$ denote the unit matrices (of order 4) for Eve's pairs and for the particles kept by Alice and Bob. Let the elements of Eve's POVM be denoted as $E^{\mu}$ (satisfying $\sum_{\mu} E^{\mu}=\mathbb{1}_{E}$ ). Then the joint state of Alice and Bob's particles, that corresponds to outcome $\mu$ registered by Eve, is the partial trace

$$
\rho_{A B}^{\mu}=\operatorname{Tr}_{E}\left[\rho\left(E^{\mu} \otimes \mathbb{1}_{A B}\right)\right]
$$

where $\rho$ is the initial state, given by Eq. (6). Note that $\rho_{A B}^{\mu}$ is not normalized: its trace is the probability that Eve observes outcome $\mu$. The above relationship readily follows from the fact that if $\left\{F^{k}\right\}$ any POVM chosen by Alice and Bob, with $\sum_{k} F^{k}=\mathbb{1}_{A B}$, then the probability of the joint result $\mu k$ is $\operatorname{Tr}\left[\rho\left(E^{\mu} \otimes F^{k}\right)\right]$.

It is not even necessary for Alice and Bob to know which experiments Eve will do. If they know nothing, they just measure $\sigma_{x}, \sigma_{y}$, or $\sigma_{z}$ (randomly chosen) on each one of their particles, instead of the specific components that appear in Eqs. (2) and (14). Later, they will learn from Eve that a definite subset of her experiments ascertained the existence of a definite entangled state of their particles. Alice and Bob don't even have to know which state this was. They will simply set apart the results of their prior measurements for the corresponding subset of particles and compute the correlations $\left\langle\sigma_{a} \otimes \sigma_{b}\right\rangle$. From the latter, they can obtain the density matrix $\rho$ of the pairs of particles in that subset [14]. Subjecting that density matrix to a partial transposition [15], they will find that the latter has a negative eigenvalue, thus verifying that the corresponding subset of particles, if it still existed, would have an entangled state.

It is obvious that from the raw data collected by Alice and Bob it is possible to select in many different ways subsets that correspond to entangled pairs. The only role that Eve has in this experiment is to tell Alice and Bob how to select such a subset. Clearly, Eve has to be honest: if she does not perform her measurements in the correct way and if she reports fake data, Alice and Bob will not select good subsets, and then their analysis will readily expose Eve's misbehaviour.

In summary, there is nothing paradoxical in the experiments outlined above. However, one has to clearly understand quantum mechanics and to firmly believe in its correctness 
to see that there is no paradox.

\section{Acknowledgments}

I am grateful to Chris Fuchs for improving the presentation of this article. This work was supported by the Gerard Swope Fund and the Fund for Encouragement of Research. 


\section{References}

[1] Bennett, C. H., Brassard, G., Crépeau, C., Jozsa, R., Peres, A., and Wootters, W. K., 1993, Phys. Rev. Lett., 70, 1895.

[2] Żukowski, M., Zeilinger, A., Horne, M. A., and Ekert, A. K., 1993, Phys. Rev. Lett., 71, 4287.

[3] Bose, S., Vedral, V., and Knight, P. L., 1998, Phys. Rev. A, 57, 822.

[4] Pan, J.-W., Bouwmeester, D., Weinfurter, H., and Zeilinger, A., 1998, Phys. Rev. Lett., 80, 3891.

[5] Braunstein, S. L., Mann, A., and Revzen, M., 1992, Phys. Rev. Lett., 68, 3259.

[6] Bennett, C. H., DiVincenzo, D. P., Smolin, J. A., and Wootters, W. K., 1996, Phys. Rev. A, 54, 3824.

[7] Hill, S., and Wootters, W. K., 1997, Phys. Rev. Lett., 78, 5022.

[8] Clauser, J. F., Horne, M. A., Shimony, A., and Holt, R. A., 1969, Phys. Rev. Lett., 23, 880.

[9] Peres, A., 1993, Quantum Theory: Concepts and Methods (Dordrecht: Kluwer).

[10] Weinfurter, H., 1994, Europhys. Lett., 25, 559.

[11] Braunstein, S. L. and Mann, A., 1995, Phys. Rev. A, 51, R1727.

[12] Michler, M., Mattle, K., Weinfurter, H., and Zeilinger, A., 1996, Phys. Rev. A, 53, R1209.

[13] Hughston, L. P., Jozsa, R., and Wootters, W. K., 1993, Phys. Lett. A 183, 14.

[14] Wootters, W. K., 1990, in Complexity, Entropy, and the Physics of Information, Santa Fe Institute Studies in the Sciences of Complexity, vol. VIII, ed. by Zurek, W. H. (Reading: Addison-Wesley).

[15] Peres, A., 1996, Phys. Rev. Lett., 77, 1413. 